\title{
Regulation of membrane-type 1 matrix metalloproteinase expression by zonula occludens- 2 in human lung cancer cells
}

\author{
E. Luczka $\cdot$ L. Syne $\cdot$ B. Nawrocki-Raby • \\ C. Kileztky $\cdot$ W. Hunziker $\cdot$ P. Birembaut . \\ C. Gilles $\cdot$ Myriam Polette
}

Received: 11 January 2013/Accepted: 8 April 2013

(C) Springer Science+Business Media Dordrecht 2013

\begin{abstract}
During tumor invasion, tumor epithelial cells acquire migratory and invasive properties involving important phenotypic alterations. Among these changes, one can observe reorganization or a loss of cell-cell adhesion complexes such as tight junctions (TJs). TJs are composed of transmembrane proteins (occludin, claudins) linked to the actin cytoskeleton through cytoplasmic adaptor molecules including those of the zonula occludens family (ZO-1, -2, -3). We here evaluated the potential role of $\mathrm{ZO}-2$ in the acquisition of invasive properties by tumor cells. In vivo, we showed a decrease of ZO-2 expression in bronchopulmonary cancers, with a preferential localization in the cytoplasm. In addition, in vitro, the localization of ZO-2 varied according to invasive properties of tumor cells, with a cytoplasmic localization correlating with invasion. In addition, we demonstrated that ZO-2 inhibition increases invasive and migrative capacities of invasive tumor cells. This was associated with an increase of MT1-MMP. These
\end{abstract}

E. Luczka · B. Nawrocki-Raby · C. Kileztky · P. Birembaut ·

M. Polette $(\square)$

INSERM UMR-S 903, SFR CAP-SANTE FED 4231, University

of Reims-Champagne-Ardenne, 45, rue Cognacq-Jay, 51100

Reims, France

e-mail: myriam.polette@univ-reims.fr

L. Syne $\cdot$ C. Gilles

Laboratory of Developmental and Tumor Biology,

GIGA-Cancer, University of Liège, Sart-Tilman,

4000 Liege, Belgium

W. Hunziker

Epithelial Cell Biology Laboratory, Institute of Molecular and Cell Biology (IMCB), Singapore 138673, Singapore

P. Birembaut · M. Polette

Laboratory of Histology, CHU of Reims, 45,

rue Cognacq-Jay, 51100 Reims, France results suggest that ZO-2, besides its structural role in tight junction assembly, can act also as a repressor of tumor progression through its ability to reduce the expression of tumor-promoting genes in invasive tumor cells.

Keywords ZO-2 - MT1-MMP · Lung cancer cells

$\begin{array}{ll}\text { Abbreviations } \\ \text { AC } & \text { Adenocarcinomas } \\ \text { C/EBP } & \text { CCAAT/enhancer-binding protein } \\ \text { CD1 } & \text { Cyclin D1 } \\ \text { DMEM } & \text { Dulbecco's modified eagle's medium } \\ \text { ECM } & \text { Extracellular matrix } \\ \text { EGF } & \text { Epidermal growth factor } \\ \text { HBECs } & \text { Human bronchial epithelial cells } \\ \text { MMP } & \text { Matrix metalloproteinase } \\ \text { MT1-MMP } & \text { Membrane-type 1 MMP } \\ \text { NES } & \text { Nuclear exporting signal } \\ \text { NLS } & \text { Nuclear localization signal } \\ \text { SAF-B } & \text { Scaffold attachment factor-B } \\ \text { SSC } & \text { Squamous cell carcinomas } \\ \text { TAZ } & \text { Transcriptional coactivator with } \quad \text { PDZ- } \\ & \text { binding motif } \\ \text { TJ } & \text { Tight junctions } \\ \text { YAP-2 } & \text { Yes kinase-associated protein 2 } \\ \text { ZASP } & \text { ZO-2 associated speckle protein } \\ \text { ZO-1 } & \text { Zonula occludens-1 } \\ \text { ZO-2 } & \text { Zonula occludens-2 } \\ \text { ZO-3 } & \text { Zonula occludens-3 }\end{array}$

Introduction

In normal epithelia, tight junctions (TJs) are multi protein complexes composed of transmembrane molecules 
(occludin, claudins and junctional adhesion molecules) linked to the actin cytoskeleton through cytoplasmic submembranous components. Among these cytoplasmic components are the zonula occludens proteins (ZO-1, ZO2, ZO-3). $\mathrm{ZO}-1$ is the oldest discovered $\mathrm{ZO}$ and is thus the most well described but data are also accumulating on ZO2 while still little is known about ZO-3. ZO-2 binds transmembrane molecules of TJs like occludin, and other cytoplasmic molecules including ZO-1 but not ZO-3. Besides its localization at the TJs, ZO-2 has also been observed in other cell compartments. ZO-2 has indeed been found to in the nuclei of sparse cultures, whereas in confluent monolayers, $\mathrm{ZO}-2$ is concentrated to at the TJs [1]. Accordingly, $\mathrm{ZO}-2$ has been more recently shown to interact with proteins that are not commonly associated to TJ, such as nuclear matrix proteins [2]. Thus, in the nucleus, ZO-2 has been described to colocalize with splicing factor SC-35, with scaffold attachment factor-B (SAF-B), and with transcription factors Jun and Fos, and CCAAT/enhancer-binding protein (C/EBP) $[1,3,4]$. The ability of ZO-2 to shuttle from the membrane to the cytosol or nucleus relies on the presence of nuclear exporting (NES) and nuclear localization (NLS) signals on its sequence $[1,2,5]$. However, the functional consequences of ZO-2 differential subcellular localization are still largely unknown.

In normal epithelia, ZO-2 is expressed as two isoforms $\mathrm{ZO}-2 \mathrm{~A}$ and $\mathrm{ZO}-2 \mathrm{C}$. $\mathrm{ZO}-2 \mathrm{C}$ protein is 23 amino acids shorter at the N-terminus than ZO-2A [6, 7]. Three others forms have also been described, two derived from form $\mathrm{A}$ and one from form $\mathrm{C}$. However little is known about the potential functional differences between these isoforms. In carcinomas, a reorganization of cell-cell junctions is frequently observed. Accordingly, ZO-2 expression has been shown to be altered in some carcinomas and a downregulation of ZO-2 expression has been reported in lung carcinomas $[8,9]$. However, in agreement with its ability to shuttle to different cell compartments, other functions, different to its structural role in TJs, have been described for ZO-2 that could contribute to modulate tumor progression. As such, ZO-2 has more particularly been implicated in the regulation of cell proliferation [10]. For example, $\mathrm{ZO}-2$ is able to suppress cell proliferation by inhibiting expression of cyclin D1 (CD1) [11, 12]. Recently, Lechuga et al. have discovered a small protein ZASP (ZO-2 Associated Speckle Protein), which interacts with ZO-2. Overexpression of ZASP blocks the inhibitory activity of ZO-2 on CD1 [13]. Also, in the nucleus, ZO-2 has been shown to bind YAP-2 (Yes kinase-associated protein 2) that is an effector of the Hippo pathway. ZO-2 thus promotes the NLS and pro-apoptotic function of YAP2 through the activation of the Hippo pathway [14]. Through the contribution of ZO-2 to cancer progression is unclear, it thus appears that it could modulate several hallmarks of cancer progression.

Besides the reorganization or the loss of cell-cell contacts, tumor invasion is also characterized by an increased ability of tumor cells to degrade extracellular matrix (ECM) components through an enhanced expression of proteases including matrix metalloproteinases (MMPs). MMPs are a family of zinc endopeptidases that are either secreted or anchored to the cell membrane. Among the membrane-type MMPs, membrane-type 1 MMP (MT1-MMP) has been particularly implicated in pericellular proteolysis associated with cell migration and invasion [15]. In numerous studies, an overexpression of MT1-MMP has been described in many types of human carcinomas [16]. In addition, several data have reported that MT1-MMP is produced by invasive tumor cells in vivo as well as in vitro and so is associated with the acquisition of an invasive phenotype by tumor cells [17]. Interestingly, a correlation between the reorganization of tight junction and the expression of MT1-MMP by epithelial tumor cells has been established [18].

In the light of these data, we here aimed at evaluating the potential role of ZO-2 in the acquisition of invasive properties by tumor cells and in the regulation of MT1MMP. We showed an association between the cytoplasmic localization of ZO-2 and the invasive phenotype of tumors cells in bronchopulmonary carcinomas and in several tumor cell lines. We also report that ZO-2 siRNA transfection in invasive cells increases their invasive properties and the expression of MT1-MMP.

\section{Materials and methods}

Tumor tissue samples

50 surgical specimens of bronchopulmonary carcinomas obtained by lobectomy and/or pneumonectomy and 25 normal lung tissues matching to the tumor samples were collected. These bronchopulmonary carcinomas included 29 squamous cell carcinomas (SSC) of stages I (7 cases), II (12 cases) and III (10 cases) and 21 adenocarcinomas (AC) of stages I ( 9 cases), II (6 cases) and III (6 cases) according to the TNM classification.

Tumor cell lines

All human lung Calu-3, 16HBE, HBE4-E6/E7, BEAS-2B, BZR cell lines used were obtained from the American Type Culture Collection (Rockville, MD, USA). Human bronchial epithelial cells (HBECs) were isolated from human normal bronchial tissues from patients undergoing surgery 
for bronchial carcinoma and were cultivated as described previously [19]. Human lung cell lines Calu-3, 16HBE, BEAS-2B, and BZR were grown in Dulbecco's modified Eagle's medium (DMEM) (Gibco, Invitrogen, Carlsbad, CA, USA) supplemented with $10 \%$ fetal calf serum (Gibco), and $100 \mathrm{UI} / \mathrm{mL}$ of penicillin and streptomycin (Gibco), and HBE4-E6/E7 (HBE4) in keratinocyte serum free medium (Gibco) supplemented with $0.2 \mathrm{ng} / \mathrm{mL}$ EGF (epidermal growth factor) and $25 \mu \mathrm{g} / \mathrm{mL}$ BPE (Bovine pituitary extract).

\section{Reverse transcription-PCR analyses}

RNA was extracted from normal and tumor lung tissues frozen in liquid nitrogen with the RNA Easy Qiagen kit (Qiagen, MD, USA) and from cells with the High Pure RNA Isolation kit (Roche Diagnostics GmbH). RT-PCR was performed with $10 \mathrm{ng}$ of total RNA by using the GeneAmp Thermostable rTth Reverse Transcriptase RNA PCR kit (Applied Biosystem, Foster City, CA, USA) and pairs of nucleotides. The reverse and forward primers (Eurogentec) for ZO-2, ZO-2A, ZO-2C, MT1-MMP, GAPDH and 28S were designed as follows : ZO-2 primers (forward $5^{\prime}-\mathrm{GCG}$ AGAAGCTGGTTTCAAGAGA-3', reverse 5'-GCTTTCG GAGTCACATCCAGTA-3'), ZO-2A primers (forward 5'-AGG-AGC-AGG-AGC-AGA-AGC-AGA-A-3', reverse 5'-CGG-GAG-CAC-ATC-AGA-AAT-GAC-A-3' $)$, ZO-2C primers (forward 5'-GCA-AGT-ACT-CCA-GGA-AGC-AC A-G-3', reverse 5'-CGG-GAG-CAC-ATC-AGA-AAT-GA C-A-3'), MT1-MMP primers (forward 5'-GGATACCCAAT GCCCATTGGCCA-3', reverse 5'-CATTGGGCATCCA GAAGAGAGC-3' ${ }^{\prime}$ ), GAPDH primers (forward $5^{\prime}$-CCAGG GCTGCTTTTAACTCTGGTA- $3^{\prime}$, reverse $5^{\prime}$-GAGGGATC TCGCTCCTGGAAGAT-3') and $28 \mathrm{~S}$ primers (Forward 5'-GTTCACCCAC TAATAGGGAACGTGA-3', Reverse 5'-GGATTCTGACTTAGAGGCGTTCAGT-3'). RT-PCR analysis was done in the linear phase of amplification by using a limited number of PCR cycles. RT-PCR products were separated by acrylamide gel electrophoresis stained with SYBR Gold (Molecular Probes) and quantified by fluorimetric scanning (LAS-1000, Fuji, Stamford, CT, USA). Data are expressed as mean \pm SEM of 3 independent in vitro experiments. A one-sample $t$ test was done for in vitro experiments and a $p$ value $<0.05$ was considered significant. A non-parametric test (Mann-Whitney $U$ test) was performed for RT-PCR analyses on lung cancer specimens and a $p$ value $<0.05$ was considered significant.

\section{Immunohistochemistry}

Immunohistochemistry was carried out on paraffin serial sections. The sections were deparaffinized, rehydrated, and treated with $3 \% \mathrm{H}_{2} \mathrm{O}_{2}$ for 20 min to quench endogenous peroxidase activity. Slides were then incubated for overnight at $4{ }^{\circ} \mathrm{C}$ with a rabbit polyclonal antibody to ZO-2 (Sigma-Aldrich, St-Louis, MO, USA) or a mouse monoclonal antibody to MT1-MMP (clone 113-B7, Oncogene Science, Cambridge, MA). After several washes, the slides were successively incubated with biotin-coupled anti-rabbit/mouse antibody (Dako, Carpinteria, CA, USA) and then with a horseradish peroxidase (HRP)-conjugated streptavidin (Dako). The peroxidase activity was revealed using AEC + (Dako). The slides were briefly counterstained with Mayer's hematoxylin, mounted, and observed under a Zeiss Axiophot microscope. Immunohistochemical detection of ZO-2 was scaled as follows: $0=$ no detection, $1=$ detection in $<10 \%$ of tumour cells, $2=$ detection in $10-25 \%$ of tumour cells, $3=$ detection in $26-50 \%$ of tumour cells, $4=$ detection in $>50 \%$ of tumour cells. Negative controls were performed by omitting the primary antibody or incubating with corresponding IgG isotype. Digestive tissue, known to express ZO-2, was used as a positive control.

Transfection of small interfering RNA

Three 19-nucleotide specific sequences were selected in the coding sequence of ZO-2 (Gene ID: 9414) to generate 21-nucleotide sense and 21-nucleotide antisense strands of the type (19 N) TT (N, any nucleotide). The sense and antisense strands were then annealed to obtain duplexes with identical $3^{\prime}$ overhangs. The sequences were submitted to a BLAST search against the human genome to ensure the specificity of the siRNA to the targeted sequence. Three corresponding scrambled duplexes, which do not recognize any sequence in the human genome, were used as controls. The 19-nucleotide specific sequences for the three ZO-2 siRNAs are as follows: ZO-2 Si1, 5'-GGUGAACACACA GGAUUUC-3'; ZO-2 Si2， 5'-GGAAACUCCACAGAG CCUG-3'; and ZO-2 Si3, 5'-GCGGUUUGGGUCUCUG AAG-3' (Eurogentec, Seraing, Belgium). For transfection of the siRNA duplexes, 100,000 cells were plated in six-well plates in $2 \mathrm{~mL} /$ well of culture medium. Twenty-four hours after plating, the cells were transfected by phosphate calcium precipitation by adding in each well $200 \mu \mathrm{L}$ of a mixture containing the a mix of three siRNA duplexes $(20 \mathrm{nmol} / \mathrm{L})$, $140 \mathrm{mmol} / \mathrm{L} \mathrm{NaCl}, 0.75 \mathrm{mmol} / \mathrm{L}$ Na2HPO4, $6 \mathrm{mmol} / \mathrm{L}$ glucose, $5 \mathrm{mmol} / \mathrm{L} \mathrm{KCl}, 25 \mathrm{mmol} / \mathrm{L}$ HEPES, and $125 \mathrm{mmol} / \mathrm{L} \mathrm{CaCl}$. Twenty-four hours after transfection, the cells were extensively washed with phosphate buffered saline (PBS) and incubated for $48 \mathrm{~h}$ in culture medium before they were harvested for reverse transcription-PCR (RT-PCR) analyses, Western blotting analyses, and migration assays. 
Transcriptomic analysis

Four micrograms of total RNA were labeled using the GeneChip ${ }^{\circledR}$ Expression $3^{\prime}$ Amplification One-Cycle Target Labeling kit (Affymetrix, Santa Clara, CA) following the manufacturer's protocol. The cRNA was hybridized to Genechip Human Genome U133 Plus 2.0 Array (Affymetrix) according to the manifacturer's protocol. Briefly, double-stranded cDNA was synthesized from $4 \mu \mathrm{g}$ of total RNA primed with a poly-(dT)-T7 oligonucteotide. The cDNA was used in an in vitro transcription reaction in the presence of T7 RNA polymerase and biotin-labeled modified nucleotides during $16 \mathrm{~h}$ at $37{ }^{\circ} \mathrm{C}$. Biotinylated cRNA was purified and then fragmented (35-200 nucleotides) together with hybridization controls and hybridized to the microarrays for $16 \mathrm{~h}$ at $45{ }^{\circ} \mathrm{C}$. Using Fluidics Station (Affymetrix), the hybridized biotin-labeled cRNA was revealed by successive reactions with streptavidin R-phycoerythrin conjugate, biotinylated anti-streptavidin antibody and streptavidin R-phycoerythrin conjugate. The arrays were finally scanned with an affymetrix/HewlettPackard GeneChip Scanner 3000 7G.

\section{Western blotting analysis}

Total protein extracts were made in RIPA buffer $(50 \mathrm{mmol} / \mathrm{L}$ Tris $\mathrm{pH} 7.4,150 \mathrm{mmol} / \mathrm{L} \mathrm{NaCl}, 1 \%$ (v/v) Igepal, Triton $1 \%, 1 \%(\mathrm{w} / \mathrm{v})$ sodium deoxycholate, $0.1 \%$ (w/v) SDS, and $5 \mathrm{mmol} / \mathrm{L}$ iodoacetamid) supplemented with complete protease inhibitor cocktail (Roche Diagnostics, Mannheim, Germany). Protein concentration was determined with the detergent-compatible protein assay (Bio-Rad). Ten microgram of total protein samples were separated by electrophoresis on $10 \%$ SDS-polyacrylamide gels and then transferred on a polyvinylidene difluoride membrane (NEN, Boston, MA, USA), which was then blocked with $5 \%$ milk (w/v), $0.1 \%$ Tween 20 (v/v) in PBS for $2 \mathrm{~h}$. The membranes were then incubated overnight at $4{ }^{\circ} \mathrm{C}$ with either a rabbit polyclonal antibody against ZO-2 (clone Z54.PL, Invitrogen) or mouse monoclonal antibody to MT1-MMP (clone 2D7, provided by Dr Rio, IGBMC, Illkirch, France). The filters were then incubated with either a HRP-conjugated goat anti-rabbit or swine antimouse secondary antibody (Dako). Signals were detected using an Enhanced Chemiluminescence Plus kit (ECL+) (Amersham Pharmacia Biotech, Buckinghamshire, United Kingdom). Subsequent detection of glyceraldehyde-3phosphate dehydrogenase (GAPDH) (clone 6C5, Chemicon, Millipore, Billerica, MA, USA) was performed on the same filters as control. Data are expressed as mean \pm SEM of 3 independent experiments. A one-sample $t$ test was done and a $p$ value $<0.05$ was considered significant.
Immunofluorescence

Confluent monolayers of cells cultured on glass coverslips were fixed with methanol for $10 \mathrm{~min}$ at $-20{ }^{\circ} \mathrm{C}$. The coverslips were then satured for 30 min with $3 \%$ BSA in PBS, and incubated with a rabbit polyclonal antibody against ZO-2 (clone Z54.PL, Invitrogen) for overnight at $4{ }^{\circ} \mathrm{C}$. After several washes, the coverslips were incubated with an Alexa fluor 594-coupled anti-rabbit IgG (Molecular Probes, Eugene, OR, USA) for $1 \mathrm{~h}$. Nuclei were stained with DAPI (Molecular Probes). The coverslips were then mounted with aquapolymount antifading solution (Polysciences Inc, Warrington, PA, USA) onto glass slides and observed under a Zeiss fluorescence microscope.

\section{Modified Boyden chamber invasion assay}

The in vitro invasive properties of cells were assessed using a modified Boyden chamber assay. A total of $10^{5}$ cells were placed in the upper compartment of the invasion chamber (BD BioCoat Matrigel Invasion Chamber; BD Biosciences, Bedford, MA, USA). For MMP inhibition experiments, GM6001 $(20 \mu \mathrm{M})($ Calbiochem, Merck Biosciences, Darmstadt, Germany) was also added to the cells in the upper compartment of the insert. The chambers were incubated for $6 \mathrm{~h}$ (BZR) and $16 \mathrm{~h}(\mathrm{BEAS}-2 \mathrm{~B})$ at $37^{\circ} \mathrm{C}$. The filters were then fixed in methanol for ten minutes and stained with hematoxylin for $5 \mathrm{~min}$. Quantification of the invasion assay was done by counting the number of cells at the lower surface of the filters (30 fields at 400 -fold magnification). Data are expressed as mean \pm SEM of 3 independent experiments. A one-sample $t$ test was done and a $p$ value $<0.05$ was considered significant.

\section{Two dimensional migration assay}

Two hours after seeding $1 \times 10^{5}$ cells/well into 12 -well plates, cell migration analysis was performed using an inverted microscope (Axiovert 200, Zeiss, Oberkochen, Germany) equipped with an environmental chamber (Incubator XL-3, Pecon, Erbach, Germany) containing $5 \%$ $\mathrm{CO}_{2}$ in air at $37^{\circ} \mathrm{C}$ and a charge-coupled device camera (Coolsnap, Roper Scientific, Photometrics, Tucson, AZ), and driven by the Axiovision software (Zeiss). Image sequences of the cells were recorded every $5 \mathrm{~min}$ for $5 \mathrm{~h}$ at $10 \times$ magnification and the trajectories and the migration speeds were analyzed with the Manual tracking ImageJ plugin (http://rsb.info.nih.gov/ij/plugins/track/track.html). Data are expressed as mean \pm SEM of 3 independent experiments. A one-sample $t$ test was done and a $p$ value $<0.05$ was considered significant. 


\section{Results}

ZO-2 delocalisation from the cell membrane correlates with a low degree of tumor differentiation

In order to compare the expression of $\mathrm{ZO}-2$ in vivo between bronchopulmonary carcinomas and normal tissues, we first carried out RT-PCR analyses to examine ZO2 mRNA expression. We found that ZO-2 is significantly less expressed in carcinomas than in normal tissues (Fig. 1A). Looking at the different ZO-2 mRNA isoforms, we found that isoform ZO-2A is reduced in SCC and AC. In contrast, $\mathrm{ZO}-2 \mathrm{C}$ mRNA expression was not altered in carcinomas. Closer examination of a possible link between ZO-2 modulation and tumor stage revealed no difference between stages I, II and III of both SCC and AC for the ZO-2 and its isoforms (Data not shown).

By immunohistochemistry, we showed that in the distant normal bronchi, ZO-2 was localized at the apical cell membrane of normal epithelial cells (Fig. 1B-a). In lung parenchyma, ZO-2 was found at the cell membrane of type II pneumocytes (Fig. 1B-d). In cancers, ZO-2 labeling was heterogeneous in localization and intensity, and some tumor clusters did not show any ZO-2 staining. In well differentiated clusters of SCC and AC, ZO-2 was detected at the cell membrane of tumor cells (Fig. 1B-b, e) whereas in poorly differentiated clusters it was present in the cytoplasm of tumor cells (Fig. 1B-c, f). Interestingly, in $\mathrm{AC}, \mathrm{ZO}-2$ was rather detected at the basal pole of the cancer cells towards the stroma or the basement membrane (Fig. 1B-e, f). ZO-2 staining was also rarely observed in the nucleus of some poorly differentiated tumor cells (data not shown). In order to address this heterogeneity of ZO-2 localization in cancers, we established a scaling method to evaluate, in each cancer specimen, the percentage of the tumor area affected by a cytoplasmic, a membranous or a nuclear staining of ZO-2 (Fig. 1B). Thus, for the majority of lung cancers, most of the tumor compartment-associated ZO-2 labeling is cytoplasmic. Only a small percentage of the tumor area $(<10 \%$ of the tumor area) displays a membranous ZO-2 staining. As for ZO-2 nuclear labeling, it can only be observed in very restricted tumor areas and in a very few number of cancers.

Our RT-PCR and immunohistochemistry results thus showed that ZO-2 expression is reduced in bronchopulmonary carcinomas and also that $\mathrm{ZO}-2$ localization varied in relationship with the dedifferentiation status of carcinomas.

The cytoplasmic localization of ZO-2 is correlated with migratory/invasive capacities of cells

To further explore a potential relationship between $\mathrm{ZO}-2$ expression and the invasive phenotype of tumor cells, we compared the expression of ZO-2 in different human lung cancer cell lines displaying various degrees of in vitro invasiveness. The invasive phenotype of these lung cancer cells, assessed by modified Boyden chamber assay, has been extensively characterized previously in our laboratory and was confirmed in the present study (Fig. 2A) [20]. Western blot analyses revealed that ZO-2 is expressed by normal human bronchial epithelial cells and by all cell lines (Fig. 2B). In addition, RT-PCR analyses showed that the two ZO-2 isoforms (A and $\mathrm{C}$ ) are also expressed by normal human bronchial epithelial cells and by all cell lines (Fig. 2C).

We next examined the subcellular localization of ZO-2 in normal human bronchial epithelial cells and in the different lung tumor cell lines by immunofluorescence microscopy. Supporting our data obtained on the tumor samples, ZO-2 was found at the cell membrane in normal human bronchial epithelial cells and in non-invasive tumor cell lines (as illustrated in HBEC and 16HBE cells in Fig. 2D-a, b, respectively), whereas it displayed a predominantly diffuse cytoplasmic and nuclear staining in invasive tumor cell lines (as illustrated in BZR cells in Fig. 2D-c).

\section{$\mathrm{ZO}-2$ regulates invasiveness of lung tumor cells}

To evaluate the potential role of $\mathrm{ZO}-2$ in tumor cell invasion, we used a RNA interference strategy to down-regulate the endogenous $\mathrm{ZO}-2$ levels in two invasive tumor cell lines (BEAS-2B and BZR). As shown in Fig. 3A, a pool of three siRNA efficiently decreased ZO-2 protein levels. We also observed a clear inhibition of the expression of the two ZO-2 mRNA isoforms in the two cells lines treated with this ZO-2 siRNA pool (Fig. 3B).

We then tested the effect of ZO-2 inhibition in lung invasive tumor cells in two models of migration assays. In the modified Boyden chamber assay, we observed a modification of invasive properties of tumor cells in vitro. Indeed, ZO-2 inhibition increased the invasive potential of BEAS-2B $\quad(2.25 \pm 0.33$ fold $)(p<0.05)$ and BZR (1.54 \pm 0.06 fold) $(p<0.05)$ cells (Fig. $3 \mathrm{C})$. In the $2 \mathrm{D}$ migration assay, trajectories of BEAS-2B and BZR transfected with ZO-2 siRNA are longer and their migration speed was significantly increased when compared to control cells $(24.73 \pm 0.12$ versus $13.47 \pm 0.12 \mu \mathrm{m} / \mathrm{h}$ and $32.87 \pm 0.82$ versus $24.09 \pm 0.0 .73 \mu \mathrm{m} / \mathrm{h}$, respectively) $(p<0.05)$ (Fig. 3D).

We also explored the effect of ZO-2 siRNA treatment on cell proliferation. ZO-2 inhibition had no effect on cell proliferation of lung cell lines in the first $48 \mathrm{~h}$ (data not shown), minimizing a potential impact of modulations of cell proliferation on the results obtained in both Boyden chamber and the 2D cell migration assays. 
A

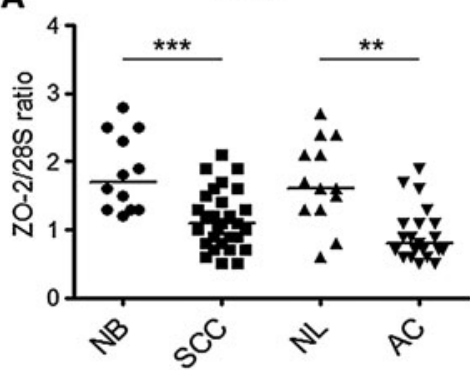

\begin{tabular}{|c|c|c|c|}
\hline Tissue & media & $\min -m$ & $p$ \\
\hline NB & 1.7 & $1.2-2.8$ & \\
\hline SCC & 1.1 & $0.5-2.1$ & 0.0007 \\
\hline $\mathrm{NL}$ & 1.6 & $0.6-2.7$ & \\
\hline$A C$ & 0.8 & $0.5-1.9$ & 0.0012 \\
\hline
\end{tabular}

B

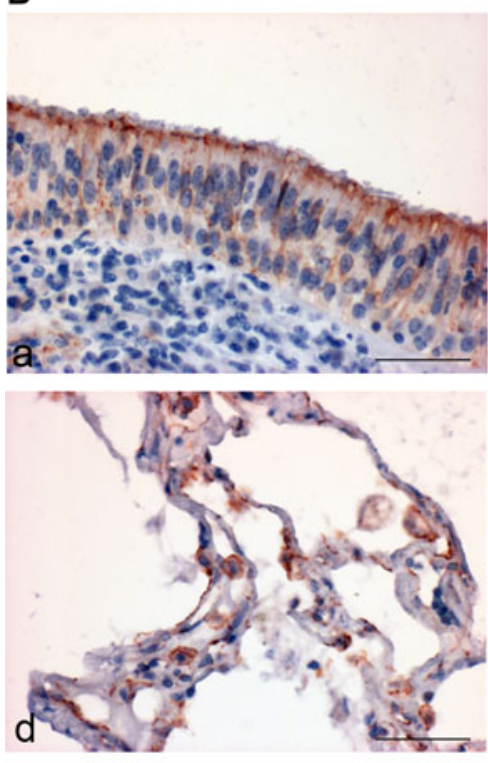

ZO-2 A

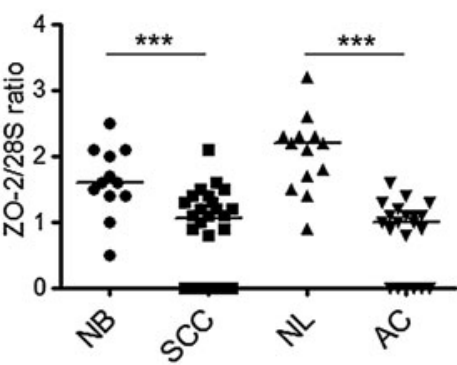

\begin{tabular}{|cccc|}
\hline Tissue & median & min-max & $\boldsymbol{p}$ \\
\hline NB & 1.6 & $0.5-2.5$ & \\
SCC & 1.05 & $0-2.1$ & 0.0006 \\
\hline NL & 2.2 & $0.9-3.2$ & \\
AC & 1 & $0-1.6$ & 0.0001 \\
\hline
\end{tabular}
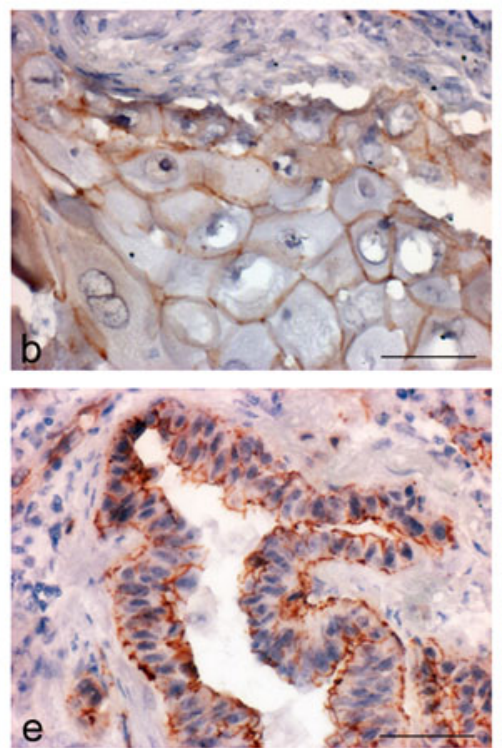

ZO-2 C

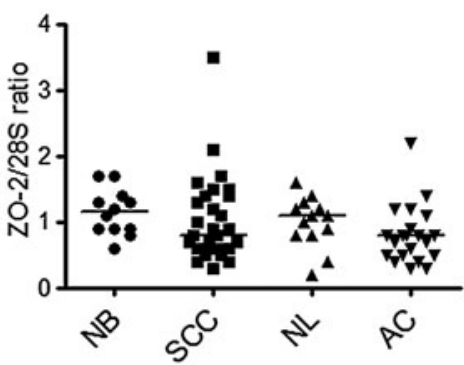

\begin{tabular}{|cccc|}
\hline Tissue & median & min-max & $\boldsymbol{p}$ \\
\hline NB & 1.15 & $0.6-1.7$ & \\
SCC & 0.8 & $0.3-3.5$ & 0.159 \\
\hline NL & 1.1 & $0.2-1.6$ & \\
AC & 0.8 & $0.3-2.2$ & 0.0747 \\
\hline
\end{tabular}
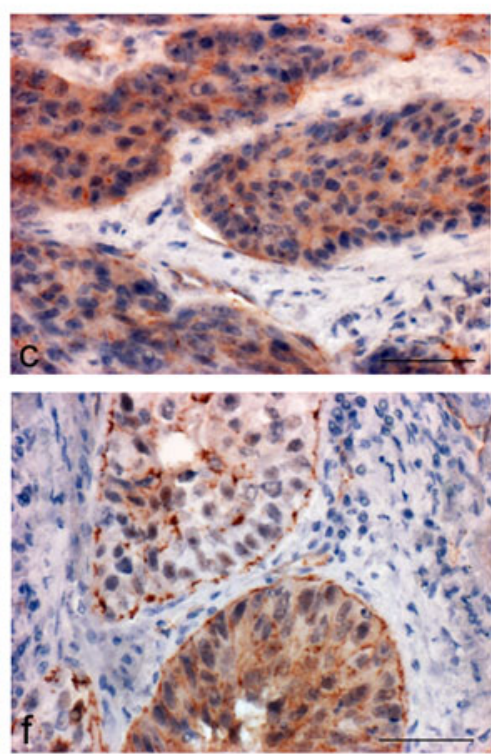

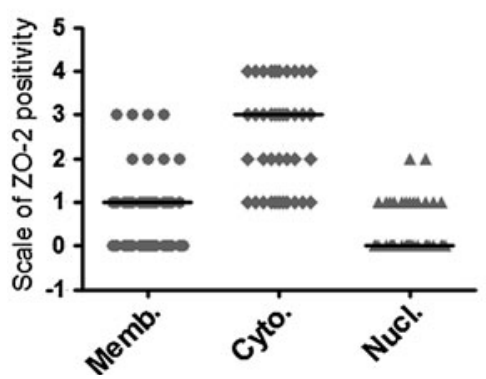

Fig. 1 ZO-2 expression in normal tissues and bronchopulmonary carcinomas. A RT-PCR analyses of ZO-2, ZO-2A and ZO-2C expression in both adenocarcinomas $(A C)$ and squamous cell carcinomas $(S C C)$ and normal tissue counterpart, normal bronchi $(N B)$ and normal lung $(N L)$, respectively. ZO-2 values have been normalized to the values obtained for 28S. Data are expressed as scatter graphs comparing the median of each group of tissues

$\mathrm{ZO}-2$ regulates MT1-MMP expression in lung invasive tumor cells

As the inhibition of $\mathrm{ZO}-2$ expression modulated the invasive properties of lung tumor cells, we aimed at identifying
$(* * p<0.01 ; * * * p<0.001)$. B ZO-2 immunostaining performed on normal bronchi and lung parenchyma $(a, d)$, on well differentiated adenocarcinomas and squamous cell carcinomas $(b, e)$ and on poor differentiated adenocarcinomas and squamous cell carcinomas $(c$, f) (bars $120 \mu \mathrm{m})$. A scaling analysis is shown, which reports the percentage of tumor area displaying membrane-associated (Memb.), cytoplasmic (Cyto.) or nuclear (Nucl.) ZO-2 staining

by $\mu$ arrays molecular factors implicated in this process that could be targeted by ZO-2 silencing. We used GeneChips Affymetrix (AffymetrixTM) to screen and compare the mRNA expression profile in invasive lung tumor BZR cells transfected with a ZO-2 siRNA and a corresponding 
A



D

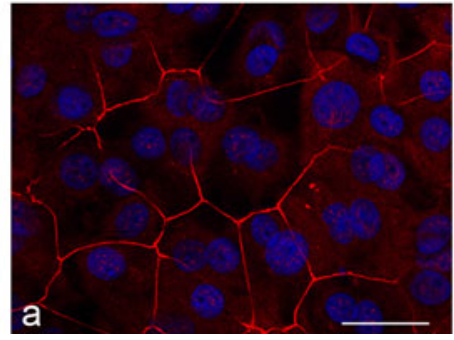

B

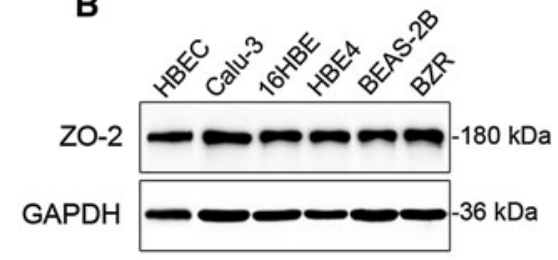

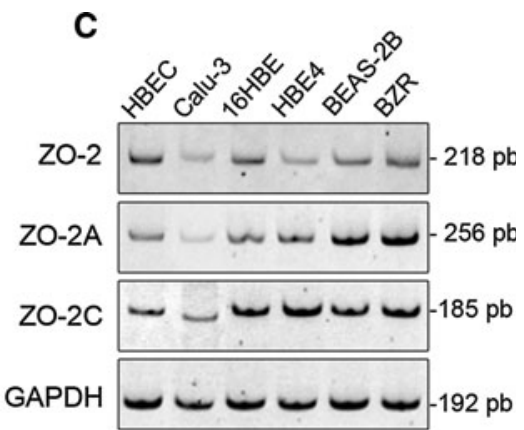

Fig. 2 Expression and localization of ZO-2 in human lung cancer cell lines displaying various degrees of in vitro invasiveness. A Analysis of the invasive capacities of human lung cell lines in a modified Boyden chamber assay. B Western blot analyses of ZO-2 and GAPDH expression in human bronchial epithelial cells (HBECs) and lung cancer cell lines. C RT-PCR analyses of ZO-2 and its isoforms expression in HBECs and in lung cancer cell lines. D Localization of ZO-2 in HBECs $(a)$, in non-invasive $(b)$ and in invasive $(c)$ tumor cell lines (16HBE and BZR, respectively) (bar $=40 \mu \mathrm{m})$ scrambled control siRNA. Among the modulated genes, MT1-MMP was strongly up-regulated following ZO-2 inhibition (Table 1; Fig. 4). This is of particular interest given the implication of MT1-MMP in invasive processes. We first confirmed the $\mu$ array results by RT-PCR and Western blotting. We observed that BEAS-2B and BZR cells transfected with ZO-2 siRNA indeed expressed more MT1-MMP mRNA $(1.68 \pm 0.08$ and $1.63 \pm 0.13$ fold respectively) ( $p<0.05$ and $p<0.01$ respectively) than their respective controls (Fig. 4A). Similarly, western blotting also revealed a significant augmentation of the $63 \mathrm{kDa}$ active form of MT1-MMP (Fig. 4B). Indeed, BEAS-2B and BZR cells transfected with ZO-2 siRNA expressed more MT1-MMP proteins than their respective controls $(2.58 \pm 0.56$ and $2.48 \pm 0.27$ fold, respectively; $p<0.05$ and $p<0.01$, respectively). Moreover, treatment of BEAS-2B and BZR cell lines with broad-spectrum synthetic MMP inhibitor GM6001 abolished siRNA ZO-2increased invasion, confirming that the pro-invasive effect of ZO-2 depletion is related to MMP activity $(p<0.001)$ (Fig. 4C).

In addition, strengthening our in vitro observations, RTPCR analyses on bronchopulmonary carcinomas and normal tissues established an inverse relationship between ZO-2 and MT1-MMP. Thus, ZO-2 was found significantly less expressed in carcinomas than in normal tissues whereas MT1-MMP was significantly more expressed in carcinomas than in normal tissues (Fig. 4D). Also, the immunodetection of MT1-MMP and ZO-2 on serial lung tumor sections allowed to observe that MT1-MMP was preferentially expressed in ZO-2-negative tumor areas (Fig. 4E). While in all cases ZO-2 negative tumor areas expressed MT1-MMP, not all MT1-MMP positive areas lacked ZO-2 staining.

\section{Discussion}

In this study, we showed a decrease of ZO-2 expression in SCC and AC as compared to normal tissues and observed a heterogenous cytoplasmic localization of ZO-2 in poorly differentiated bronchopulmonary carcinomas. In addition, in vitro, we demonstrated that the localization of $\mathrm{ZO}-2$ varies according to invasive/migratory properties of tumor cells. Interestingly, we further demonstrated that ZO-2 inhibition increases invasive capacities of tumor cells in association with enhanced expression of MT1-MMP.

First, we observed that mRNA levels of ZO-2 are reduced in human bronchopulmonary carcinomas as compared to normal lung tissues. In agreement with our findings, previous data have reported a decrease of ZO-2 in tumor tissues of breast and lung when compared to normal tissue [8,9]. These data are in agreement with the notion that the expression of tight junction proteins is down 

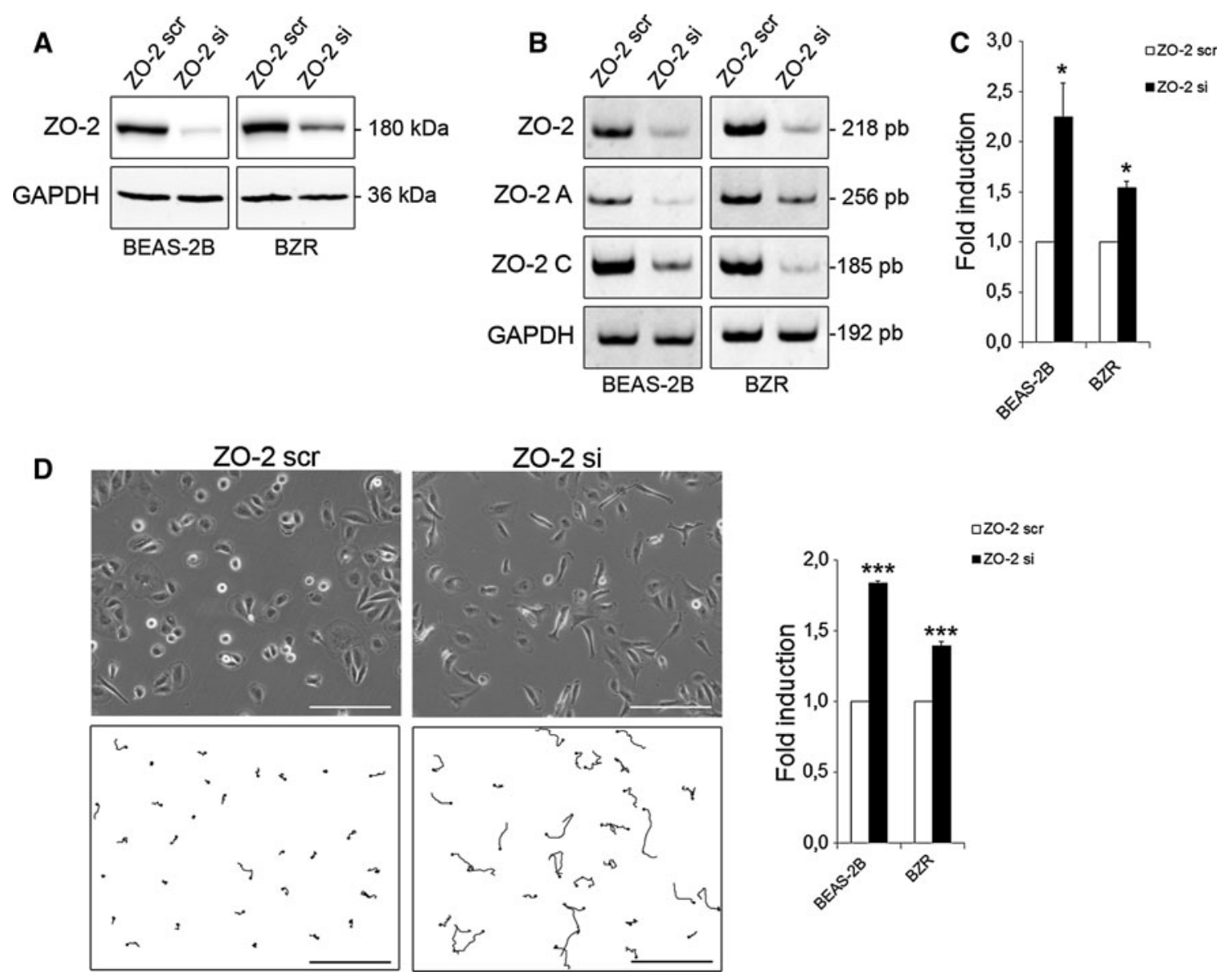

Fig. 3 Effect of ZO-2 siRNA on in vitro cell invasion of invasive lung tumor cells. A Western blot analyses of ZO-2 and GAPDH expression on total extracts of BEAS-2B and BZR cells transfected ZO-2 siRNA (ZO-2 si) or scrambled siRNA (ZO-2 scr). B RT-PCR analyses of ZO-2, ZO-2 isoforms (ZO-2A and ZO-2C) and GAPDH expression in BEAS-2B and BZR cells transfected with ZO-2 siRNA (ZO-2 si) or scrambled siRNA (ZO-2 scr). C Boyden chamber

regulated in carcinomas [21]. Complementing these observations, we have shown a significant decrease of the $\mathrm{ZO}-2 \mathrm{~A}$ isoform in lung tumors, while the $\mathrm{ZO}-2 \mathrm{C}$ isoform showed no difference as compared to normal tissue. The literature regarding the two described $\mathrm{ZO}-2$ isoforms is extremely scarce and reports that the expression of specific ZO-2 isoforms is dependent on the cell type suggest different biological functions for these two molecules [7]. In agreement with our observations, Chlenski et al. [6] have not detected isoform $\mathrm{A}$ in pancreatic adenocarcinoma when compared to normal tissues, while isoform $\mathrm{C}$ was detected in all tumor samples. Thus, our results suggest that a diminution of the ZO-2A form could be specifically involved in the process of tumor progression of bronchopulmonary carcinomas.

In addition to a decrease of ZO-2 expression in bronchopulmonary carcinomas, our results showed a different subcellular localization of this molecule in tumor cells as analyses of invasive properties of ZO-2 siRNA (ZO-2 si) -transfected cells compared to scrambled control siRNA (ZO-2 scr)-transfectants $(* p<0.05)$. D Phase-contrast photographs and trajectories of BEAS2B transfected with scrambled (ZO-2 scr) or ZO-2 siRNA (ZO-2 si) in a two-dimensional migration assay (bars $120 \mu \mathrm{m}$ ). Comparison of migration speeds of BEAS-2B and BZR transfected with scrambled (ZO-2 scr) or ZO-2 siRNA (ZO-2 si) $(* * * p<0.001)$

compared to normal lung epithelial cells. Indeed, ZO-2 is localized at the apical cell membrane of normal lung epithelial cells and in the cytoplasm and/or nucleus of poorly differentiated tumor cells. Similarly, Martin et al. [22] have reported that ZO-2 displayed a cytoplasmic staining in infiltrating tumor cells of breast cancer, whereas it was found at the cell membrane of normal mammary gland epithelial cells. Moreover, we also observed that in well differentiated tumors clusters of SCC and AC, ZO-2 was detected at the cell membrane of the tumor cells, whereas in poorly differentiated tumor clusters, it was present in the cytoplasm of the tumor cells. Taken together, these data suggest that ZO-2 relocalization from the cell membrane to the cytoplasm/nucleus is associated with the dedifferentiation status of carcinomas. Emphasizing these in vivo data, our in vitro data shows an association between a cytoplasmic localization of ZO-2 and high migratory/invasive capacities of tumor cell lines. Indeed, ZO-2 is present at the 
Table 1 Analysis by GeneChips Affymetrix of mRNA profiles in BZR cells transfected with siRNA against ZO-2 compared with corresponding scrambled siRNA

\begin{tabular}{|c|c|c|c|}
\hline Genes & siZO-2/scrZO-2 & Genes & siZO-2/scrZO-2 \\
\hline CTSF & 5.98 & ETV6 & 0.49 \\
\hline MYB & 5.97 & UBE2H & 0.49 \\
\hline IRF7 & 4.1 & IL1B & 0.49 \\
\hline CDKL3 & 3.86 & MGST1 & 0.49 \\
\hline FOSB & 3.55 & STAT5B & 0.49 \\
\hline CASP3 & 3.49 & HMGB 1 & 0.48 \\
\hline $\mathrm{CTSH}$ & 3.47 & FOXP1 & 0.47 \\
\hline RRM2 & 3.17 & NQO1 & 0.46 \\
\hline TLE2 & 3.08 & SMAD2 & 0.45 \\
\hline ADAM9 & 2.94 & NOTCH2 & 0.45 \\
\hline TP53 & 2.94 & ITGA10 & 0.44 \\
\hline ZEB2 & 2.83 & STAT3 & 0.44 \\
\hline MCM7 & 2.83 & CASP6 & 0.43 \\
\hline CCNE2 & 2.56 & TJP2 (2) & 0.43 \\
\hline CDKN2C & 2.5 & PPIC & 0.43 \\
\hline HMGB2 & 2.45 & PSME3 & 0.43 \\
\hline CCNE1 & 2.45 & UBE2J2 & 0.42 \\
\hline MT1-MMP & 2.43 & E2F5 & 0.41 \\
\hline $\mathrm{CCNY}$ & 2.37 & MYBL1 & 0.40 \\
\hline TIMP2 & 2.33 & KRT7 & 0.39 \\
\hline DVL1 & 2.32 & MAPK9 & 0.39 \\
\hline CDCA1 & 2.30 & CDK6 & 0.38 \\
\hline $\mathrm{E} 2 \mathrm{~F} 2$ & 2.30 & TNFSF10 & 0.37 \\
\hline PKMYT1 & 2.29 & IGF2BP3 & 0.36 \\
\hline TK1 & 2.27 & ARRB1 & 0.36 \\
\hline TGFB 1/1 & 2.22 & TNFSF14 & 0.33 \\
\hline MAP4K3 & 2.22 & NOTCH1 & 0.33 \\
\hline UBE2Z & 2.22 & ITGB7 & 0.32 \\
\hline ETV4 & 2.18 & PECAM1 & 0.27 \\
\hline MAP2K7 & 2.10 & RHOB & 0.26 \\
\hline CDK10 & 2.03 & PPID & 0.26 \\
\hline MDM1 & 2.03 & LDHA & 0.25 \\
\hline $\mathrm{CCNG} 2$ & 2.02 & TJP2 (1) & 0.20 \\
\hline PSMB9 & 2.01 & KRT81 & 0.15 \\
\hline PSME3 & 0.50 & IL1A & 0.11 \\
\hline HMGN4 & 0.49 & CAPN6 & 0.04 \\
\hline
\end{tabular}

cell membrane of normal bronchial epithelial cells and of lung non-invasive cells and is located in the cytoplasm and/ or nucleus of lung invasive cell lines. Previously, Islas et al. [1] have demonstrated that $\mathrm{ZO}-2$ was found at the membrane in MDCK confluent cell monolayers, while it was present in the nucleus in sparse cells. Taken together, these data support the general concept that $\mathrm{ZO}-2$ relocalization from the membrane to the cytoplasm/nucleus could be associated with dedifferentiation and increased migration/invasive properties of epithelial tumor cells.
Interestingly, we observed an upregulation of the proinvasive gene MT1-MMP in response to ZO-2 siRNA transfection in invasive tumor cell lines. Moreover, we also showed that ZO-2 inhibition induces an increase of invasive capacities of these cell lines. All the data suggest that cytoplasmic and/or nuclear ZO-2, even though associated with dedifferentiation and invasive status of tumor cells, could remain a negative regulator of invasive processes. These latest observations suggest different function between ZO-1 and ZO-2. Indeed, we and others have shown an association between a cytoplasmic/NLS of ZO-1 and an invasive/migratory behavior of different cell lines $[18,23,24]$. However, in contrast with our observations on $\mathrm{ZO}-2$, decreasing ZO-1 by RNA interference in invasive cell lines was shown to decrease both MT1-MMP expression and invasive properties [18]. In the same line of thinking, Reichert et al. reported a stimulation of EMT when overexpressing a truncated form of $\mathrm{ZO}-1$ that is less prone to associate to TJs [25]. This EMT promoting effect has been associated with an activation of the beta catenin pathway. The actual belief regarding ZO-1 is thus that cytoplasmic/nuclear pools of ZO-1 would rather have an oncogenic activity. This is thus contrasting with our current findings on ZO-2, suggesting an anti-tumoral role of ZO-2 independently of its subcellular localization.

The mechanisms by which ZO-2 may regulate tumor invasion remain to be determined. A first hypothesis would be that ZO-2 could act directly as a repressor of pro-invasive transcription factors. Indeed, previous studies have shown that ZO-2 is able to colocalize with several proteins binding nucleic acids such as SC-35 factor, SAF-B factor, and to associate with the transcription factors Fos, Jun and CCAAT/ enhancer binding protein $[1,3,4]$. In addition, recent work showed that ZO-2 negatively regulates the transcriptional function of TAZ (Transcriptional coactivator with PDZbinding motif), a factor implicated in the promotion of epithelial-mesenchymal transition and tumor progression [2628]. Another mean by which ZO-2 could regulate tumor invasion is by modifying the shuttling between different subcellular compartments of factors regulating tumor invasion-modulating genes. For example, the involvement of ZO-2 in modulating the Hippo tumor suppressor pathway through its interaction with YAP-2 (Yes kinase-associated protein-2) has recently been uncovered. Association of YAP2 with ZO-2 allows its localization to the nucleus and thus increases its pro-apoptotic function [14, 29]. In addition to YAP-2, ZO-2 can also serve as a shuttle to deliver ARVCF into the nucleus $[14,30]$. Thus, the absence or the diminution of ZO-2 expression, as we mimicked by siRNA transfection, could have a significant impact on the different functions of these factors. In this context, $\mathrm{ZO}-2$ could be involved in different signaling activities modulating invasion-associated target genes. 

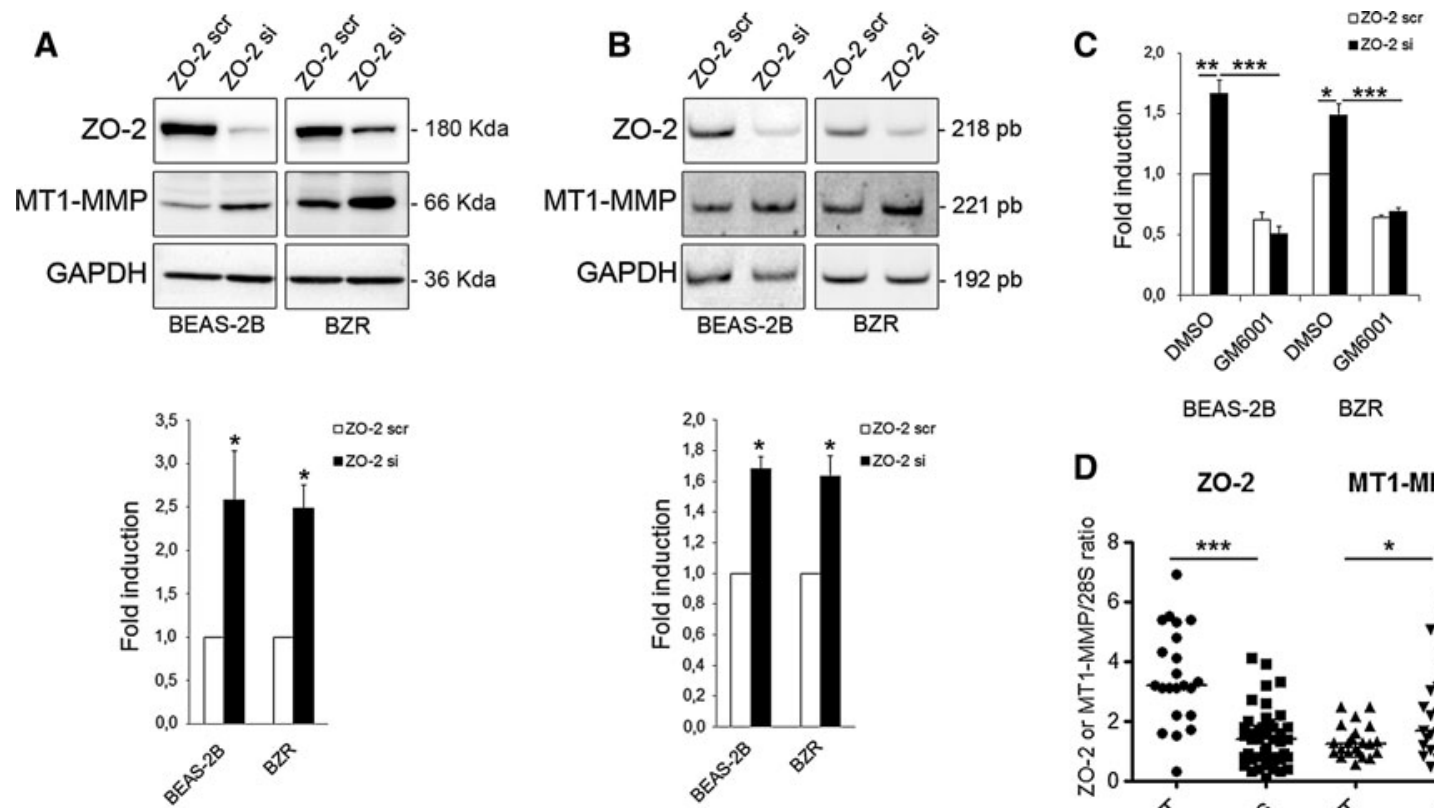

E
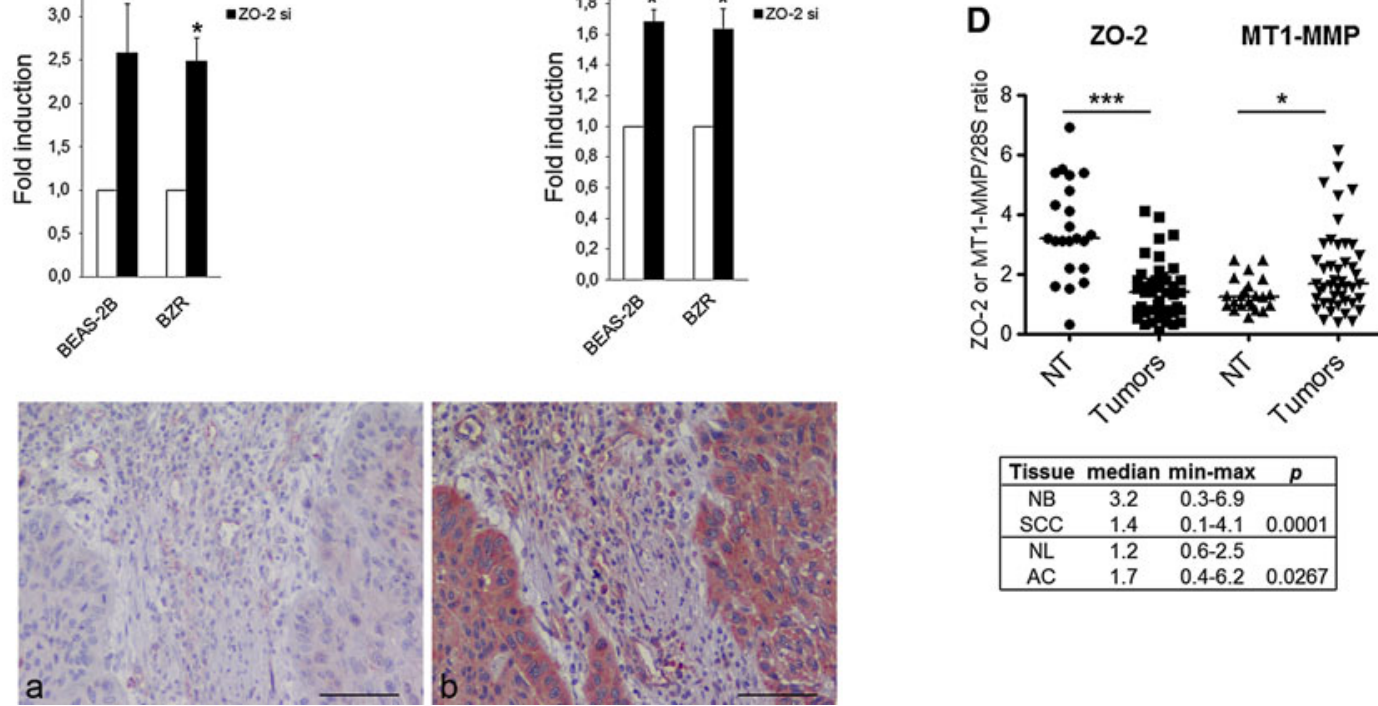

Fig. 4 Up-regulation of MT1-MMP expression in invasive lung tumor cells by ZO-2 siRNA. A Western blot analyses of ZO-2, MT1MMP and GAPDH expression on total extracts of BEAS-2B and BZR cells transfected with siRNA against ZO-2 (ZO-2 si) or scrambled siRNA (ZO-2 scr) $(* p<0.05)$. B RT-PCR analyses of ZO-2, MT1MMP and GAPDH expression in BEAS-2B and BZR transfected cells with siRNA against ZO-2 (ZO-2 si) or scrambled siRNA (ZO-2 scr) $(* p<0.05)$. C Boyden chamber analyses of invasive properties of BEAS-2B and BZR cells transfected with a siRNA against ZO-2 (ZO-

In conclusion, our data show that ZO-2, even when not associated with adhesion complexes, can negatively modulate tumor invasion through its ability to downregulate the expression of pro-invasive genes such as MT1-MMP.

Acknowledgments The research effort associated with this article was funded in part by the "Région Champagne-Ardenne", the "Ligue Contre le Cancer", the Lions's Clubs of Soissons, Villers-Cotterets and Crépy en Valois, Un Euro contre le Cancer and the Fonds National pour la Santé ACI 2004-2010 INCa (Cancéropôle Grand-Est project), the Fonds de la Recherche Scientifique-FNRS (FRS-FNRS, Belgium), the "Partenariat Hubert Curien-Tournesol".

\section{References}

1. Islas S, Vega J, Ponce L et al (2002) Nuclear localization of the tight junction protein ZO-2 in epithelial cells. Exp Cell Res 274:138-148
2 si) or a scrambled siRNA (ZO-2 scr) and treated with GM6001 $(20 \mu \mathrm{M})$ or DMSO as a control $(* * * p<0.001 ; * * p<0.01$; $* p<0.05)$. D RT-PCR analyses of ZO-2 and MT1-MMP expression in lung carcinomas (Tumors) and normal tissue (NT). ZO-2 and MT1MMP values were normalized to 28S. Data are expressed as scatter graphs comparing the median of each group of tissues $(* * * p<0.001$; $* p<0.05$ ). $\quad$ E Immunohistochemistry of ZO-2 (a) and MT1MMP (b) in the same tumor area of serial sections in a poorly differentiated squamous cell carcinomas (bar $80 \mu \mathrm{m})$

2. Jaramillo BE, Ponce A, Moreno J et al (2004) Characterization of the tight junction protein $\mathrm{ZO}-2$ localized at the nucleus of epithelial cells. Exp Cell Res 297:247-258

3. Traweger A, Fuchs R, Krizbai IA et al (2003) The tight junction protein ZO-2 localizes to the nucleus and interacts with the heterogeneous nuclear ribonucleoprotein scaffold attachment factorB. J Biol Chem 278:2692-2700

4. Betanzos A, Huerta M, Lopez-Bayghen E et al (2004) The tight junction protein ZO-2 associates with Jun, Fos and C/EBP transcription factors in epithelial cells. Exp Cell Res 292:51-66

5. Gonzalez-Mariscal L, Ponce A, Alarcon L et al (2006) The tight junction protein ZO-2 has several functional nuclear export signals. Exp Cell Res 312:3323-3335

6. Chlenski A, Ketels KV, Tsao MS et al (1999) Tight junction protein ZO-2 is differentially expressed in normal pancreatic ducts compared to human pancreatic adenocarcinoma. Int $\mathrm{J}$ Cancer 82:137-144

7. Chlenski A, Ketels KV, Korovaitseva GI et al (2000) Organization and expression of the human zo-2 gene (tjp-2) in normal and neoplastic tissues. Biochim Biophys Acta 1493:319-324 
8. Paschoud S, Bongiovanni M, Pache JC et al (2007) Claudin-1 and claudin-5 expression patterns differentiate lung squamous cell carcinomas from adenocarcinomas. Mod Pathol 20:947-954

9. Tokes AM, Szasz AM, Juhasz E et al (2012) Expression of tight junction molecules in breast carcinomas analysed by array PCR and immunohistochemistry. Pathol Oncol Res 18:593-606

10. Gonzalez-Mariscal L, Bautista P, Lechuga S et al (2012) ZO-2, a tight junction scaffold protein involved in the regulation of cell proliferation and apoptosis. Ann N Y Acad Sci 1257:133-141

11. Huerta M, Munoz R, Tapia R et al (2007) Cyclin D1 is transcriptionally down-regulated by $\mathrm{ZO}-2$ via an $\mathrm{E}$ box and the transcription factor c-Myc. Mol Biol Cell 18:4826-4836

12. Tapia R, Huerta M, Islas S et al (2009) Zona occludens-2 inhibits cyclin D1 expression and cell proliferation and exhibits changes in localization along the cell cycle. Mol Biol Cell 20:1102-1117

13. Lechuga S, Alarcon L, Solano J et al (2010) Identification of ZASP, a novel protein associated to Zona occludens-2. Exp Cell Res 316:3124-3139

14. Oka T, Remue E, Meerschaert K et al (2010) Functional complexes between YAP2 and ZO-2 are PDZ domain-dependent, and regulate YAP2 nuclear localization and signalling. Biochem $\mathrm{J}$ 432:461-472

15. Polette M, Nawrocki-Raby B, Gilles C et al (2004) Tumour invasion and matrix metalloproteinases. Crit Rev Oncol Hematol 49:179-186

16. Seiki M, Mori H, Kajita M et al (2003) Membrane-type 1 matrix metalloproteinase and cell migration. Biochem Soc Symp 70:253-262

17. Gilles C, Newgreen D, Sato H, Thompson EW (2005) Matrix metalloproteases and epithelia-to-mesenchymal transition: implications for carcinoma metastasis. In: Savagner P (ed) Rise and fall of epithelial phenotype: concepts of epithelial-mesenchymal transition. Eurekah.com and Kluwer Academic/Plenum Publishers, New York, pp 1-19

18. Polette M, Gilles C, Nawrocki-Raby B et al (2005) Membrane-type 1 matrix metalloproteinase expression is regulated by zonula occludens-1 in human breast cancer cells. Cancer Res 65:7691-7698

19. Lesimple P, van SI, Buisine MP et al (2007) Trefoil factor family 3 peptide promotes human airway epithelial ciliated cell differentiation. Am J Respir Cell Mol Biol 36:296-303
20. Toupance S, Brassart B, Rabenoelina F et al (2012) Elastinderived peptides increase invasive capacities of lung cancer cells by post-transcriptional regulation of MMP-2 and uPA. Clin Exp Metastasis 29:511-522

21. Martin TA, Jiang WG (2009) Loss of tight junction barrier function and its role in cancer metastasis. Biochim Biophys Acta 1788:872-891

22. Martin TA, Watkins G, Mansel RE et al (2004) Loss of tight junction plaque molecules in breast cancer tissues is associated with a poor prognosis in patients with breast cancer. Eur J Cancer 40:2717-2725

23. Gottardi CJ, Arpin M, Fanning AS et al (1996) The junctionassociated protein, zonula occludens-1, localizes to the nucleus before the maturation and during the remodeling of cell-cell contacts. Proc Natl Acad Sci USA 93:10779-10784

24. Polette M, Mestdagt M, Bindels S et al (2007) Beta-catenin and ZO-1: shuttle molecules involved in tumor invasion-associated epithelial-mesenchymal transition processes. Cells Tissues Organs 185:61-65

25. Reichert M, Muller T, Hunziker W (2000) The PDZ domains of zonula occludens-1 induce an epithelial to mesenchymal transition of Madin-Darby canine kidney I cells. Evidence for a role of beta-catenin/Tcf/Lef signaling. J Biol Chem 275:9492-9500

26. Chan SW, Lim CJ, Guo K et al (2008) A role for TAZ in migration, invasion, and tumorigenesis of breast cancer cells. Cancer Res 68:2592-2598

27. Lei QY, Zhang H, Zhao B et al (2008) TAZ promotes cell proliferation and epithelial-mesenchymal transition and is inhibited by the hippo pathway. Mol Cell Biol 28:2426-2436

28. Remue E, Meerschaert K, Oka T et al (2010) TAZ interacts with zonula occludens- 1 and -2 proteins in a PDZ-1 dependent manner. FEBS Lett 4(19):4175-4180

29. Oka T, Schmitt AP, Sudol M (2011) Opposing roles of angiomotin-like-1 and zona occludens-2 on pro-apoptotic function of YAP. Oncogene 31(1):128-134

30. Kausalya PJ, Phua DC, Hunziker W (2004) Association of ARVCF with zonula occludens (ZO)-1 and ZO-2: binding to PDZdomain proteins and cell-cell adhesion regulate plasma membrane and nuclear localization of ARVCF. Mol Biol Cell 15: $5503-5515$ 